\title{
Editorial
}

\section{Thermodynamics and Heat Transfer in Fires}

Since the dawn of time fire has been shedding light on the progress of mankind, first as a destructive supernatural entity to be scared of, then as an irreplaceable source of energy for technological progress as well as for domestic life. In this schematic picture, the myth of Prometeus is a beautiful metaphor of the transition between the two ages.

Despite the impressive technological advancement since those times, today fire can still represent a major hazard when it develops without a strict control, and fire safety has become an important concern in many aspects of our everyday life. Thus, there is a significant effort of the scientific community to understand the dynamics of fires as well as their consequences on the surrounding environment.

This Hot Topic Issue of the Open Thermodynamics Journal contains five contributions reporting some recent developments of Fire Science and Engineering, on topics of particular technical and social relevance.

Fires are complex phenomena, resulting from several physical and chemical processes characterised by a multiplicity of length and time scales. Length scales range from the nanometres when addressing material flammability to the kilometres when dealing with forest fires, while time scales cover a broad spectrum ranging from the several hours necessary to heat structural elements, to the few milliseconds required to complete most chemical reactions. Thus, it is important to establish general scaling laws, which are reviewed by Simeoni and Torero in the context of forest fires, universally recognised as a major natural hazard. In particular, they introduce a general model for flaming ignition of solid fuel, discussing the scaling of laboratory experimental results, and extend the results to modelling combustion in forest fires.

A series of catastrophic fire accidents in European road and railway tunnels occurred in the recent past (Mount Blanc, 1999; Tauern, 1999; Gotthard 2001; Frejus 2005) raised public awareness about fire safety in tunnels, and triggered a significant research effort in tunnel fire safety and risk analysis. Cafaro and Bertola review the development of fire safety research in road and railway tunnels during the past 20 years, with particular reference to the activity of the Flame Research Group at Politecnico di Torino (Italy). These results provide a basis to develop rational design criteria and safety regulations.

Among the various safety and fire mitigation devices used in tunnels, water mist systems represent an emerging technology, which has attracted significant attention in the past few years. Santangelo and Tartarini present a comprehensive review of the existing literature about water-mist systems, with particular attention to water mist characterization and its performance in fire control and mitigation. It turns out that whilst these systems are very promising in terms of efficiency and potential in fire control and suppression, they still require much work to reach the status of standard technology.

Another classical topic in fire research is the study of compartment fires, i.e. those fires occurring in a closed environment, such as a room, for which several deterministic as well as stochastic models have been proposed in the open literature. In particular, deterministic models can be sorted into zone models, which return the main parameters of the fire as the result of first-order ordinary differential equations, and field models, which simulate the fire evolution within extended geometric domains using general-purpose CFD packages, using specific sub-models for buoyancy, radiation heat transfer, and turbulence. While most of these models are essentially based on a thermal energy balance, Bertola and Cafaro propose to study compartment fires taking into account the limitations of the Second Law of Thermodynamics. In other words, in this approach the standard energy balance is completed by the condition of non-negative entropy generation for the system under consideration.

Finally, Bennardo and Inzaghi present a mathematical model to calculate the evolution in time of the smoke during a compartment fire, and to estimate the time needed for smoke to fill the compartment, as a function of the thermophysical properties of the flame and of the compartment geometry. The model also allows one to calculate the volumetric flow rate and the enthalpy flow of the smoke through the openings in the building envelope, which are directly related to the environmental impact of the fire.

\author{
Volfango Bertola \\ (Guest Editor) \\ University of Edinburgh, School of Engineering, \\ The King's Buildings, Mayfield Road, Edinburgh EH9 3JL, \\ UK \\ Tel: +44-131-6508697 \\ Fax: +44-131-6506551 \\ E-mail: V.Bertola@ed.ac.uk
}

(C) V. Bertola; Licensee Bentham Open.

This is an open access article licensed under the terms of the Creative Commons Attribution Non-Commercial License (http://creativecommons.org/licenses/by-nc/3.0/) which permits unrestricted, non-commercial use, distribution and reproduction in any medium, provided the work is properly cited. 